\title{
Microembolism from aortic aneurysm and ventricular thrombus: a complication of intravenous streptokinase
}

\author{
John S. Abraham, Moira Wilson'1, Viorel Scripcariu, Peter C. Barnes ${ }^{1}$ and \\ Roger W. Marcuson
}

University Departments of Surgery and 'Medicine, Hope Hospital, Eccles Old Road, Salford, Manchester M6 $8 H D, U K$

\begin{abstract}
Summary: Two patients sustained the rare complication of skin infarction following administration of intravenous streptokinase for acute myocardial infarction. We report evidence that dissolution of thrombus from an unsuspected source and subsequent microembolization of the skin may be responsible for this complication. In patients known to have an aortic aneurysm or ventricular thrombus, careful consideration should be given to the use of intravenous streptokinase following myocardial infarction.
\end{abstract}

\section{Introduction}

Intravenous streptokinase is widely used for the treatment of acute myocardial infarction and has been associated with an improved patient survival. ${ }^{1}$ We report the rare complication of skin necrosis following the use of intravenous streptokinase. Evidence is given that the aetiology of the infarction is microembolism from an unsuspected source of thrombus in the left ventricle or an aortic aneurysm. These cases demonstrate the potential microembolic events that may occur following the use of thrombolytic agents in such patients.

\section{Case reports}

Case 1

A 65 year old man presented as an emergency with severe central chest pain of 2 hours duration. He had sustained two myocardial infarctions 9 and 7 years previously, which had not been treated with thrombolytic therapy. Bilateral lower limb amputations had been performed 5 years previously for atherosclerotic peripheral vascular disease. Medication at presentation included aspirin, frusemide and isosorbide mononitrate. Examination revealed him to be hypertensive $(200 / 100 \mathrm{mmHg})$ with no clinical cardiac failure or palpable aortic aneurysm. Inferior myocardial infarction was confirmed by electrocardiograms and subsequently elevation of serum cardiac enzymes. One and a half million units of streptokinase (Kabikinase ${ }^{\mathrm{R}}$ ) were infused

Correspondence: R.W. Marcuson, F.R.C.S.

Accepted: 9 March 1994 intravenously via an antecubital fossa vein. An intravenous infusion of glyceryl trinitrate was used to control his hypertension.

Three hours following infusion of streptokinase, the patient complained of lower abdominal pain and backache. There was fixed purple discoloration of the skin in the right groin, thigh and over the penis (Figure 1). Blood pressure and pulse rate were stable and haemoglobin estimation was unaltered from admission levels. Because the differential diagnosis of abdominal pain included ruptured aortic aneurysm, ultrasonography and computerized tomography (CT) of the abdomen was performed, revealing an aortic aneurysm with a maximum transverse diameter of $4.3 \mathrm{~cm}$. However, there was no evidence of retroperitoneal or intraabdominal haemorrhage to suggest rupture.

The skin lesions demarcated along the line of fixed staining and necrosed over the following

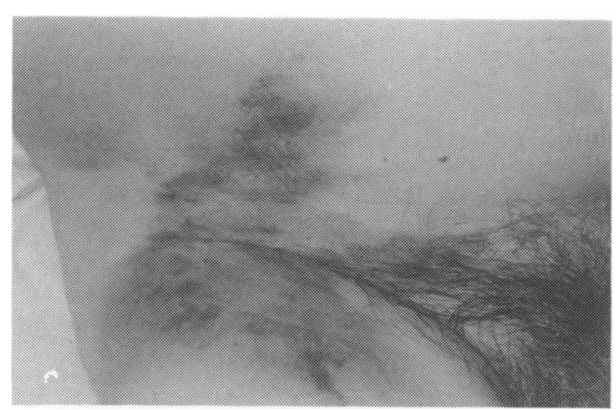

Figure 1 Case 1: skin changes in the right groin 3 hours following administration of one and a half million units of intravenous streptokinase. 
week. The abdominal and back pain gradually settled and bowel function remained normal. He was discharged home 16 days following myocardial infarction. Three months later the skin lesions had healed spontaneously with no apparent sequelae.

\section{Case 2}

A 60 year old man presented as an emergency with central chest pain radiating to the left arm, of one hour duration. He had sustained an anterolateral myocardial infarction with left ventricular mural thrombus 7 years previously. This had not been treated with thrombolytic therapy but he had been anti-coagulated for 3 months following infarction. Current medication included metoprolol, aspirin and bezafibrate. Examination revealed no evidence of cardiac failure or aortic aneurysm and electrocardiogram showed changes of an acute inferolateral myocardial infarction. One and a half million units of streptokinase (Kabikinase ${ }^{R}$ ) were infused intravenously via an antecubital fosa vein.

Six hours following streptokinase the patient experienced epigastric and back pain, and was tender in the epigastrium. There was purple, fixed staining of the skin overlying the lower abdomen, buttocks (Figure 2) and thighs, and similar changes in the right foot. Femoral and distal pulses were all palpably normal. There was no evidence of aortic aneurysm on CT of the abdomen. A two-dimensional echocardiogram suggested mural thrombus in the apex of the left ventricle.

Skin biopsy of the left thigh was performed in order to exclude vasculitis as a cause for the renal failure. This showed discrete foci of full thickness epidermal necrosis, subepidermal blistering and necrosis of underlying superficial dermis. Inflammation was virtually absent and there was thromboembolus present within a small vessel in the reticular dermis (Figure 3).

The patient developed acute renal failure with oliguria, haematuria and worsening biochemical



Figure 2 Case 2: skin changes over the back and buttocks 6 hours following administration of one and a half million units of intravenous streptokinase.

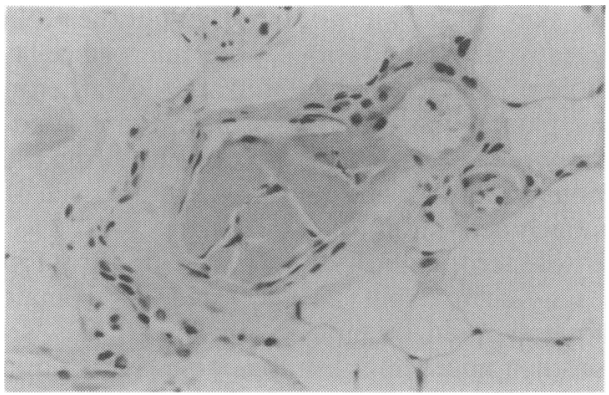

Figure 3 Case 2: occlusion of a small dermal blood vessel by thromboembolus (haematoxylin and eosin, objective $\times 40)$.

renal function (serum urea maximum $35.6 \mathrm{mmol} / \mathrm{l}$, serum creatinine maximum $512 \mu \mathrm{mol} / \mathrm{l})$. The patient made an otherwise uncomplicated recovery from the myocardial infarction. At 6 weeks following infarction the skin lesions over the buttocks and thighs had spontaneously epithelialized and healed. The hallux of the right foot became gangrenous and required amputation. Renal function gradually returned to normal.

\section{Discussion}

The patients reported above had no specific contraindications to thrombolytic therapy, yet both sustained a severe complication that we think was related to the streptokinase infusion.

Symptoms of abdominal and back pain, together with skin changes, occurred approximately 6 hours after streptokinase administration. Erythematous skin changes associated with acute anaphylaxis may occur following intravenous streptokinase. ${ }^{2}$ However, the clinical course and histological changes of the skin in case 2 were more typical of infarction than anaphylaxis. The changes in the foot of case 2 were similar to those seen in the thromboembolic phenomenon of 'trash foot' occasionally observed following aortic surgery. We believe that the aetiology of this skin necrosis is microembolic, due to dissolution of adherent thrombus from either the heart or the wall of an aortic aneurysm. This is supported by the histological findings in the skin biopsy from case 2 . The presence of thromboembolus within a small vessel in the reticular dermis suggests that a 'shower' of microemboli was responsible for the observed skin infarction.

Stafford et al ${ }^{13}$ reported seven cases of suspected systemic embolism following intravenous anistreplase or streptokinase. Skin mottling of the lower limbs occurred in four patients and skin infarction in one patient. Histological evidence of microem- 
bolic skin lesions was not reported but the authors suggested that the lesions were microembolic in aetiology. Cholesterol embolization of atheromatous plaque has previously been implicated in peripheral and visceral infarction following intravenous streptokinase, ${ }^{4.5}$ or intravenous tissue plasminogen activator. ${ }^{6}$

Cholesterol embolization following thrombolysis may arise from an ulcerated atheromatous plaque exposed by clot dissolution. If this is the case, then microembolism by cholesterol and microthrombus represent two pathological features of the same process. Thrombolytic agents may also lyse left atrial thrombus leading to large vessel occlusion. ${ }^{7}$

Lower back pain following streptokinase or anistreplase may be attributed to an allergic phenomenon. ${ }^{8.9}$ Both our cases described abdominal and back pain, and epigastric tenderness. Abdominal and lower back pain may be symptomatic of visceral ischaemia due to microembolism. Arpesani et al. ${ }^{10}$ have described mobilization of atheromatous material from the wall of an aortic aneurysm leading to fatal infarction of viscera and the lower extremities. Case 2 developed acute renal failure with haematuria. The only apparent aetiological factor to account for this is a microembolic phenomenon secondary to thrombolysis. We believe that the skin biopsy supports this thesis.

Occult microembolization may occur more frequently than previously realized. Pulmonary

\section{References}

1. ISIS-2 (Second International Study of Infarct Survival) Collaborative Group. Randomised trial of intravenous streptokinase, oral aspirin, both, or neither among 17,187 cases of suspected myocardial infarction: ISIS-2. Lancet 1988, ii: $349-360$.

2. Bednarczyk, E.M., Sherlock, S.C., Farah, M.G. \& Green, J.A. Anaphylactic reaction to streptokinase with first exposure: case report and review of the literature. Drug Intell Clin Pharm 1989, 23: 869-872.

3. Stafford, P.J., Strachan, C.J.L., Vincent, R. \& Chamberlain, D.A. Multiple microemboli after disintegration of clot during thrombolysis for acute myocardial infarction. Br Med J 1989, 299: $1310-1312$.

4. Schwartz, M.W. \& McDonald, G. Cholesterol embolization syndrome. Occurrence after intravenous streptokinase therapy for myocardial infarction. JAMA 1987, 258: 1934- 1935.

5. Pirson, Y., Honhon, B., Cosyns, J.P. \& van Ypersele, C. Cholesterol embolism in a renal graft after treatment with streptokinase. Br Med J 1988, 296: 394-395. emboli occur during thrombolysis of deep venous thromboses, " but there are few reports of clinically apparent systemic arterial emboli during intravenous thrombolysis. Nevertheless, our experience suggests that it may be advisable to attempt to exclude possible sources of emboli prior to streptokinase administration. In most cases abdominal palpation will be adequate to detect large aortic aneurysms. Abdominal ultrasonography is not recommended, since this would lead to delay in the administration of thrombolytic agents. Similarly, the use of routine echocardiography prior to thrombolysis is not recommended, unless there is a previous history of ventricular thrombus and echocardiography is readily available.

In summary, the cases we have reported suggest that arterial microemboli and skin infarction may occur when aneurysmal or ventricular thrombus is lysed by intravenous streptokinase. In our cases this complication was not fatal but increased the morbidity following myocardial infarction. In patients with an abdominal aortic aneurysm or ventricular thrombus, careful consideration should be given to the use of intravenous streptokinase following myocardial infarction.

\section{Acknowledgement}

The authors are grateful to Dr I. Roberts, Department of Pathology, Hope Hospital, Salford, for his help in preparation and interpretation of the histological slides.

6. Arora, R.R., Magun, A.M., Grossman, M. \& Katz, J. Cholesterol embolization syndrome after intravenous tissue plasminogen activator for acute myocardial infarction. $\mathrm{Am}$ Heart J 1993, 126: 225-228.

7. Blazer, D., Degroat, T., Kotler, M.N. et al. Peripheral embolization during thrombolytic therapy for left atrial thrombus. Am J Cardiol 1986, 58: 554-555.

8. Shah, M. \& Taylor, R.T. Low back pain associated with streptokinase (letter). Br Med J 1990, 301: 1219.

9. Hannaford, P. \& Kay, C.R. Back pain and thrombolysis (letter). Br Med J 1992, 304: 915.

10. Arpesani, A., Beretta, L., Giorgetti, P.L., Varesi, C. \& Matturri, L. Atheromatous material mobilization syndrome after thrombolytic therapy with streptokinase in acute myocardial infarct. Minerva Cardioangiol 1991, 39: 251-254.

11. Holmstrom, M., Bratt, G., Tornebohm, E., Rhedin, A.S. \& Lockner, D. Fatal pulmonary embolism caused by streptokinase treatment of deep venous thrombosis of the leg? $J$ Intern Med 1990, 228: 647-649. 